\title{
Laterality of Static and Dynamic Balance Abilities during One-leg Standing
}

\author{
Hiroki Aoki ${ }^{1, *}$, Shinichi Demura ${ }^{2}$, Hiroshi Hirai ${ }^{3}$ \\ ${ }^{1}$ National Institute of Technology, Fukui College, General course, Fukui, Japan \\ ${ }^{2}$ Kanazawa University, Ishikawa, Japan \\ ${ }^{3}$ Osaka Prefecture University, Osaka, Japan \\ *Corresponding author: aoki@fukui-nct.ac.jp
}

\begin{abstract}
Lower human limbs may not show laterality, differing from preferential use of upper limbs, because both legs are generally used at the same time. This study examined laterality of static and dynamic balance abilities during one-leg standing. The subjects were 100 healthy male university students (age $19.6 \pm 2.4$ years, height 172.3 $\pm 6.2 \mathrm{~cm}$, weight $64.8 \pm 8.5 \mathrm{~kg}$ ). All subjects were judged right-leg dominant based on a previous survey. They underwent static and dynamic balance tests with each lower limb. A total path length during one-leg standing on a fixed stabilometer for the static balance test and an omnidirectional stability index during one-leg standing on an unstable platform (DYJOC Board) for the dynamic balance test was each used as an evaluation parameter. The mean of two trials was used as a representative value in each test. Intraclass correlation coefficients (ICCs) in both balance tests were very high $(\mathrm{ICC}=0.75-0.91)$. A non-significant difference between means of dominant and non-dominant legs was found in both tests; their correlations were significant and high (0.93 and 0.75$)$. In addition, a correlation between dynamic and static balance tests in both legs was found be significant but low (0.21-0.25). In conclusion, the laterality is not found in the static and dynamic balance abilities during one-leg standing evaluated by the tests selected in this study, and the relation between both abilities is negligible size.
\end{abstract}

Keywords: balance abilitie, one-leg standing, laterality

Cite This Article: Hiroki Aoki, Shinichi Demura, and Hiroshi Hirai, "Laterality of Static and Dynamic Balance Abilities during One-leg Standing." American Journal of Sports Science and Medicine, vol. 6, no. 1 (2018): 11-14. doi: 10.12691/ajssm-6-1-3.

\section{Introduction}

Balance ability is divided largely into static and dynamic. Static balance ability is exerted to maintain a stable stationary posture, and dynamic balance ability is exerted to maintain stable posture during active movements such as walking, running, or recovering a posture [1]. Humans need greater balance ability exertion during one-leg standing than during both-legs standing to maintain stable posture because of a small supporting base area and an imposing large load on one leg. Although human limbs appear anatomically symmetrical, they do show laterality. Kawabata et al. [2] examined laterality of upper limbs using the following tests related to activities of daily living: the pegboard test (Buddenberg \& Davis, 2000), the moving beans with tweezers test [3], and the pursuit rotor test [4]. Many researchers [4,5] reported that laterality is found in grip strength of dominant and non-dominant hands with different use frequency in daily life. On the other hand, regarding the lower limb, Grouios et al. [6] reported a dominant leg used for maneuvering a ball and the other used for maintaining postural stability. However, Burnie and Brodie [7], Neumann et al. [8], and Masuda et al. [9] reported that a right-left difference was not found in maximum extension strength of hip and knee joints.
Matsuda et al. [10] reported that a simple one-leg balance test with low difficulty does not show clear laterality. Lower limbs are little used dominantly; mostly, they are used similarly, such as when walking. Hence, lower limbs may not show laterality or a marked difference, as do upper limbs.

Until now, laterality of balance ability has been examined by one-leg standing [11] and center of foot pressure (COP) tests during one-leg standing [10]. These tests evaluate static balance ability. On the other hand, dynamic balance ability has been evaluated by movement tasks using both legs, for instance, Timed "Up \& Go" and Sit-to-Stand tests $[12,13]$. Namely, a test using only one leg has not been developed; in addition to difficulty, none has been needed. Hence, lower limbs' laterality of dynamic balance ability has not been examined. Recently, however, a balance board with incorporated acceleration sensors has been developed. This device can quantify the degree and rate of sway and has been used to evaluate dynamic balance ability $[14,15,16]$. Dynamic balance ability coping with a disturbance load can be related to the balance board test because it is performed on an unstable board (passive stimulation). This test is possible even in a one-leg standing test, which assumes that balance ability during one-leg standing on an unstable board differs from that during one-leg standing on a stable floor. Thus, this study examined laterality of static and dynamic balance abilities during one-leg standing and their relationship. 


\section{Methods}

\subsection{Subjects}

Subjects were 100 healthy male university students (age $19.6 \pm 2.4$ years, height $172.3 \pm 6.2 \mathrm{~cm}$, weight $64.8 \pm 8.4 \mathrm{~kg}$ ). All subjects were confirmed to be right-leg dominant based on a questionnaire developed by Sato et al. [17]. Before testing, this study's aims and procedures were explained to them in detail, and written informed consent was obtained. This experimental protocol was approved by the Ethics Committee on Human Experimentation of Faculty of Human Science, Kanazawa University (approval number: 2012-03).

\subsection{Experimental Instruments and Methods}

The instrument used for the static balance test was the Anima stabilometer G5600 (Anima Corp., Tokyo, Japan). Subjects placed the supporting leg's foot on the board's center, matching landmarks of $\mathrm{X}$ - and $\mathrm{Y}$-axes marked on the board and the other foot (non-supporting leg) on the ground. The subject lifted the non-supporting leg after the tester's signal and stood on one leg for the test's duration. This device can calculate center of pressure (COP) of vertical loads from readings of three vertical load sensors, located in corners of an isosceles triangle on a level surface. Data sampling frequency was $20 \mathrm{~Hz}$. In a previous study [1], persons with larger total trace length and change velocity of COP were evaluated as "inferior" in postural control ability. Hence, participants with smaller total trace length in this study were judged "superior" in postural stability.

The instrument used for the dynamic balance test was the Dyjoc Board Plus (SV-200; SAKAI Med, Tokyo, Japan). Subjects placed the supporting leg's foot on the board's center and the other foot (non-supporting leg) on the ground. They lifted the non-supporting leg after the tester's signal and stood on one leg during the test. A keel-shaped base is attached to the back central part of a flat board that can slant up to $12^{\circ}$ backward and forward and $7^{\circ}$ side to side. This device can simultaneously measure the anteroposterior and lateral inclination angles relative to the horizontal plane using a built-in accelerometer with a sampling rate of $40 \mathrm{~Hz}$. Data are sent directly to a personal computer for analysis using Dyjoc Board Plus software (SV-200; Sakai Med). Acceleration changes used to measure the subject's balance ability are calculated using angle variations in the $\mathrm{X}$-axis (anteroposterior direction) and the Y-axis (lateral direction). The omnidirectional stability index (OSI) was selected as an evaluation parameter. Participants with smaller value were judged "superior" in postural stability.

Before performing each test, participants were instructed to watch a circular target placed at eye level during the test, without touching the lifted leg to the supporting leg. Tests were conducted two times with a 1-min rest between trials. At random, participants selected the balance test and the use order of dominant and non-dominant legs. During the test when they fell from the board or released a hand from the waist, they performed the test again. A mean of two trials was used as a representative value for each test.

\subsection{Statistical Analysis}

Trial-to-trial reliability was examined using intraclass correlation coefficients (ICCs). A t-test was used to examine the mean difference between groups. Relationships between parameters were examined using Pearson's correlation coefficient. The level of statistical significance was set at 0.05 .

\section{Results}

The ICC of the static balance test was 0.90 in the dominant leg and 0.91 in the non-dominant leg; that of the dynamic balance test was 0.76 in the former and 0.75 in the latter. No significant difference was found between means of dominant and non-dominant legs in the balance tests (Table 1). Correlations between both legs in both tests were significant and high (static balance test, 0.93; dynamic balance test, 0.75) (Table 2). In addition, a correlation between dynamic and static balance tests in both legs was found to be significant but low (0.21-0.25).

Table 1. Mean, SD, ICC, and t-value of static and dynamic one-leg balance tests

\begin{tabular}{|c|c|c|c|c|c|c|c|c|c|}
\hline & & \multicolumn{2}{|c|}{$1 \mathrm{st}$} & \multicolumn{2}{|c|}{$2 \mathrm{st}$} & \multicolumn{4}{|c|}{ Representative value } \\
\hline & & Mean & SD & Mean & $\mathrm{SD}$ & ICC & Mean & SD & t-value \\
\hline Static balance test & Dominant & 138.3 & 50.9 & 127.0 & 45.6 & 0.90 & 132.7 & 47.5 & 0.17 \\
\hline (Total trace length $(\mathrm{cm}))$ & Non-dominant & 138.0 & 52.8 & 127.9 & 44.3 & 0.91 & 133.0 & 47.9 & \\
\hline Dynamic balance test & Dominant & 408.4 & 110.0 & 383.3 & 110.4 & 0.76 & 395.8 & 104.1 & 1.77 \\
\hline$\left(\operatorname{OSI}\left({ }^{\circ}\right)\right)$ & Non-dominant & 429.9 & 131.6 & 389.8 & 114.5 & 0.75 & 409.9 & 116.9 & \\
\hline
\end{tabular}

Table 2. Correlations of each parameter

\begin{tabular}{|c|c|c|c|c|}
\hline & 1 & 2 & 3 & 4 \\
\hline 1 Dominant Total trace length & 1.00 & & & \\
\hline 2 Non-dominant Total trace length & $0.93 *$ & 1.00 & & \\
\hline 3 Dominant OSI & $0.25^{*}$ & $0.24 *$ & 1.00 & \\
\hline 4 Non-dominant OSI & 0.17 & $0.21 *$ & $0.75^{*}$ & 1.00 \\
\hline
\end{tabular}

$* \mathrm{p}<0.05$. 


\section{Discussion}

Balance ability is important for humans to maintain stable posture. COP sway during standing has a close relationship with static balance ability $[18,19,20]$ and sway characteristics from infants to the elderly have been studied [21,22]. In addition, until now, Timed "Up \& Go" and Sit-to-Stand tests have been used to evaluate dynamic balance ability $[12,13]$. This study examined laterality of static and dynamic balance abilities and their relationship in the lower limb.

That the one-leg balance test has high reliability has been clarified [23,24]. The present study found no significant difference between trial means in all tests, and ICCs were very high $(>0.76)$. Hence, tests in this study were judged to have high reliability.

No significant difference was found between means of dominant and non-dominant legs for evaluation parameters (total trace length and omnidirectional stability index) in static and dynamic balance tests. The one-leg balance test requires mainly posture control through ankle and hip joints, regardless of difference in static and dynamic balance tests. According to Kouzaki and Masani [25], the plantar flexor muscles participate in plantar flexion of the ankle, and body sway in the anteroposterior direction increases with a decrease of plantar strength. The gluteus medius muscle related to the abductor of the hip joints makes the pelvis stabilize, and the quadriceps femoris muscle related to extension of knee joints contributes their fixation [26]. It was reported that the maximum strength of these abductors and extensors does not show a right and left difference $[7,8,9,26]$. According to Lin and Woollacott's report [27], balance ability is affected by leg strength. In the case of one-leg standing, leg strength, in addition to posture control ability, contributes greatly to achieve $\mathrm{COP}$ and balance board tests. However, as mentioned above, there is no right-left difference in leg strength. Hence, it is inferred that no right-left difference is found in static and dynamic balance abilities.

In addition, relationships between dominant and nondominant legs were high in both balance tests. From present results, it seems that the relationship between exertion values of right and left legs in static and dynamic balance tests is high and that there is no evidence of laterality. Until now, Iwasaki and Yamamoto [28] reported that a close relationship between forefoot movements during walking is found in the right and left legs. Because both legs are seldom dominantly used in daily life, the relationship between both legs in both tests may have been high. In addition, relationships between static balance and dynamic balance tests were low in both legs. Static balance may differ from dynamic balance in spite of the same balance ability.

The static balance test based on one-leg standing is performed by fixing the supporting foot, such as one-leg standing time test and Functional Reach test, etc. In the general dynamic balance test, both legs are drifting, but in the present balance test, the supporting leg was fixed on the board, and the board itself fluctuated (passive stimulation). Future research should examine the lower limb's laterality using the dynamic balance test with active stimulation.

\section{Conclusion}

In conclusion, the laterality is not found in static and dynamic balance abilities during one-leg standing, and the relation between both abilities is negligible size.

\section{References}

[1] Aoki H, Demura S, Kawabata H, Sugiura H, Uchida Y, Xu N, Murase H. (2012). Evaluating the effects of open/closed eyes and age-related differences on center of foot pressure sway during stepping at a set tempo. AAR, 1(3), 72-77.

[2] Kawabata H, Demura S, Kitabayashi T, Shin S, Sato S. (2012). Gender and the laterality of various coordination tests. Gazz Med Ital, 171, 135-141.

[3] Shin S, Demura S, Kim M. (2008). Examination of practice effect and laterality in the Purdue pegboard and moving beans with tweezers tests. The Journal of Education and Health Science, 54(2), 123-128.

[4] Noguchi T, Demura S, Nagasawa Y, Uchiyama M. (2009). Influence of measurement order by dominant and nondominant hands on performance of a pursuit-rotor task. Percept Mot Skills, 108, 905-914.

[5] Aoki H, Demura S. (2008). Characteristics and lateral dominance of hand grip and elbow flexion powers in young male adults. $J$ Physiol Anthropol, 27, 201-206.

[6] Grouios G, Hatzitaki V, Kollias N, Koidou I. (2009). Investigating the stabilising and mobilising features of footedness. Laterality, 14, 362-380.

[7] Burnie J, Brodie DA. (1986). Isokinetic measurement in preadolescent males. Int J Sports Med, 7(4), 205-209.

[8] Neumann DA, Soderberg GL, Cook TM. (1988). Comparison of maximal isometric hip abductor muscle torques between hip sides. Phys Ther, 68(4), 496-502.

[9] Masuda K, Kikuhara N, Takahashi H, Yamanaka K. (2003). The relationship between muscle cross-sectional area and strength in various isokinetic movements among soccer players. J Sports Sci, 21(10), 851-858.

[10] Matsuda S, Demura S, Demura T. (2010). Examining differences between center of pressure sway in one-legged and two-legged stances for soccer players and typical adults. Percept Mot Skills, 110(3), 751-760.

[11] Hahn T, Foldspang A, Vestergaard E, Ingemann-Hansen T. (1999) One-leg standing balance and sports activity. Scand J Med Sci Sports, 9(1), 15-18.

[12] Podsiadlo D, Richardson S. (1991). The timed "Up and Go": a test of basic functional mobility for frail elderly persons. J Am Geriatr Soc, 39(2), 142-148.

[13] Yamada T, Demura S. (2004). Influence of the relative difference in chair seat height according to different lower thigh length on floor reaction force and lower-limb strength during sit-to-stand movement. J Physiol Anthropol Appl Human Sci, 23, 197-203.

[14] Kawabata H, Demura S, Uchiyama M. (2013). Effects of differences of manipulation and supporting legs and moving target speed on a visual tracking test using center of pressure. Advances in Physical Education, 3(4), 205-208.

[15] Noguchi T, Demura S, Nakagawa T. (2013). Posture stability during one-leg stance on an unstable moving platform and its relationship with each leg. Percept Mot Skills 116(2), 555-563.

[16] Ogaya S, Ikezoe T, Soda N, Ichihashi N. (2011). Effects of balance training using wobble boards in the elderly. J Strength Cond Res, 25(9), 2616-2622.

[17] Sato S, Demura S. (2012). Characteristics of the subjective, stabilization and mobilization preference leg: the relationship between these preference legs and the leg used for stabilization and mobilization activities. Gazz Med Ital, 171(2), 123-130.

[18] Demura S, Kitabayashi T, Kimura A, Matsuzawa J. (2005). Body sway characteristics during static upright posture in healthy and disordered elderly. J Physiol Anthropol Appl Human Sci, 24(5), 551-555.

[19] Demura S, Kitabayashi T, Noda M. (2006). Selection of useful parameters to evaluate center-of-foot pressure movement. Percept Mot Skills, 103(3), 959-973. 
[20] Noda M, Demura S, Kitabayashi T, Imaoka K. (2005) Examination of quantitative and fractal analysis of sway characteristics of the center of foot pressure movement during a static upright posture: Analysis based on alcohol intake, J Sports Med Phys Fitness, 45(2), 229-237.

[21] Fujiwara K, Ikegami H, Okada M Koyama Y. (1982). Contribution of age and muscle strength of lower limbs to steadiness and stability in standing posture. Anthropol Sci, 90(4), 385-399.

[22] Demura S, Kitabayashi T, Noda M, Aoki H. (2008). Age-stage differences in body sway during a static upright posture based on sway factors and relative accumulation of power frequency. Percept Mot Skills, 107(1), 89-98.

[23] Giorgetti MM, Harris AB, Jette A. (1998). Reliability of clinical balance outcome measures in the elderly. Physiother Res Int, 3, 274-283.
[24] Curb DJ, Ceria-Ulep DC, Rodriguez LB, Grove J, Guralnik J, Willcox JB, Donlon AT, Masaki HK, Chen R. (2006). Performance-based measures of physical function for highfunction populations. J Am Geriatr Soc, 54, 737-742.

[25] Kouzaki M, Masani K. (2012). Postural sway during quiet standing is related to physiological tremor and muscle volume in young and elderly adults. Gait Posture, 35(1), 11-17.

[26] McCurdy K, Langford G. (2005). Comparison of unilateral squat strength between the dominant and non-dominant leg in men and women. J Sports Sci Med, 4, 153-159.

[27] Lin SI, Woollacott M. (2005). Association between sensorimotor function and functional and reactive balance control in the elderly. Age Ageing, 34(4), 358-363.

[28] Iwasaki Y, Yamamoto S. (2011). Three-dimensional motion analysis of the foot during gait focusing on the movement of the lower leg, calcaneus and forefoot. Rigakuryoho Kagaku, 26, 371-375. 